Präv Gesundheitsf 2021 · 16:263-268

https://doi.org/10.1007/s11553-020-00815-8

Eingegangen: 5. Juni 2020

Angenommen: 23. September 2020

Online publiziert: 21. Oktober 2020

๑) Der/die Autor(en) 2020

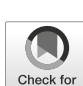

\title{
Suzanne Lischer
}

Hochschule Luzern, Soziale Arbeit, Luzern, Schweiz

\section{Die Inanspruchnahme von präventiven Angeboten durch Migrantinnen und Migranten mit glücksspielspezifischen Problemen}

Zugangsbarrieren für Migrantinnen und Migranten mit glücksspielbedingten Problemen in Bezug auf die Inanspruchnahme von präventiven Angeboten bestehen und mit welchen Strategien die Wahrscheinlichkeit der Inanspruchnahme ebendieser Angebote erhöht werden kann.

\section{Methode} ethnischen Gruppen im Vergleich zu der Mehrheitsgesellschaft höher ist [12]. Dabei nehmen Personen mit Migrationshintergrund keineswegs häufiger an Glücksspielen teil als die einheimische Bevölkerung. Doch sind diejenigen, die an Glücksspielen teilnehmen, möglicherweise gefährdeter, Probleme zu entwickeln [14, 29].

\section{Hintergrund und Fragestellung}

Befunden zufolge suchen pathologische Spielende mit Migrationshintergrund seltener Beratungs- und Behandlungsstellen auf [2, 4]. Die chancengerechte Ausrichtung von Präventions- und $\mathrm{Be}$ handlungsangeboten erfordert es, dass Zugangsbarrieren für Personen mit Migrationshintergrund mit glückspielbedingten Problemen möglichst gering sind. Entsprechend stellt sich die Frage nach der Ausgestaltung von präventiven Maßnahmen, die sich gezielt an die entsprechenden Personengruppen richten [16]. Im Rahmen einer Literaturrecherche wird die Frage untersucht, welche
Die Literatursuche fand im Zeitraum zwischen Juli und August 2019 statt. Davon ausgehend, dass richtungsweisende Studien in die aktuellen Studien Eingang gefunden haben, wurde auf aktuelle Studien ab dem Jahr 2014 Bezug genommen. Für die Literatursuche wurden die Datenbanken PubMed, Embase und Google Scholar ausgewählt. Für die Literaturrecherche wurden Schlüsselbegriffe und Wortstämme für die vier Beschreibungsdimensionen „Gambling“, „Migrant“, „Prevention“ und „Help-Seeking“ festgelegt. Davon ausgehend, dass richtungsweisende Studien in die aktuellen Studien Eingang gefunden haben, wurde auf aktuelle Studien ab dem Jahr 2014 Bezug genommen (• Abb. 1).

Die Datenextraktion erfolgt unter Verwendung der PRISMA-Richtlinien [21] Insgesamt ergab die Suche 1049 Artikel. Bei diesen Artikeln wurden die Titel geprüft. 3 weitere Artikel wurden identifiziert, indem die Bibliografien der identifizierten Artikel durchsucht wurden. In das Ereignisprotokoll wurden diejenigen Publikationen aufgenommen, welche einen direkten Bezug zum Glücksspiel hatten $(n=447)$. Bei diesen Artikeln wurde der Abstract gelesen. Letztlich wurden 38 Artikel als geeignet eingestuft und gelesen. Eingeschlossen wurden nur Studien, welche explizit die Dimensionen „Migrant“, „Inanspruchnahme von präventiven Angeboten“ sowie "glücksspielsuchtbedingte Probleme" beinhalteten. Artikel, welche die Inanspruchnahme von Maßnahmen des Verbraucherschutzes wie bspw. die Spielersperre behandeln, wurden nicht berücksichtigt. Gemäß der Ein- und Ausschlusskriterien und nach Prüfung auf Eignung wurden 12 Studien aufgenommen (- Tab. 1).

\section{Ergebnisse}

\section{Zugangsbarrieren}

Generell sind die Raten der Inanspruchnahme von Präventions- und Behandlungsangeboten bei pathologischen Spielenden niedrig $[4,17,19,27]$. Die Gründe dafür sind vielfältig: Scham, die Angst vor Stigmatisierung, das Leugnen des Problems, die Überzeugung, es selber lösen zu können, und die Angst vor hohen Behandlungskosten scheinen die wesentlichen Barrieren darzustellen [11]. Mehrere Studien legen nahe, dass die Angst vor Stigmatisierung bei Personen mit Migrationshintergrund besonders ausgeprägt ist $[5-10,18,20,22,24,29]$. In der Mi- 


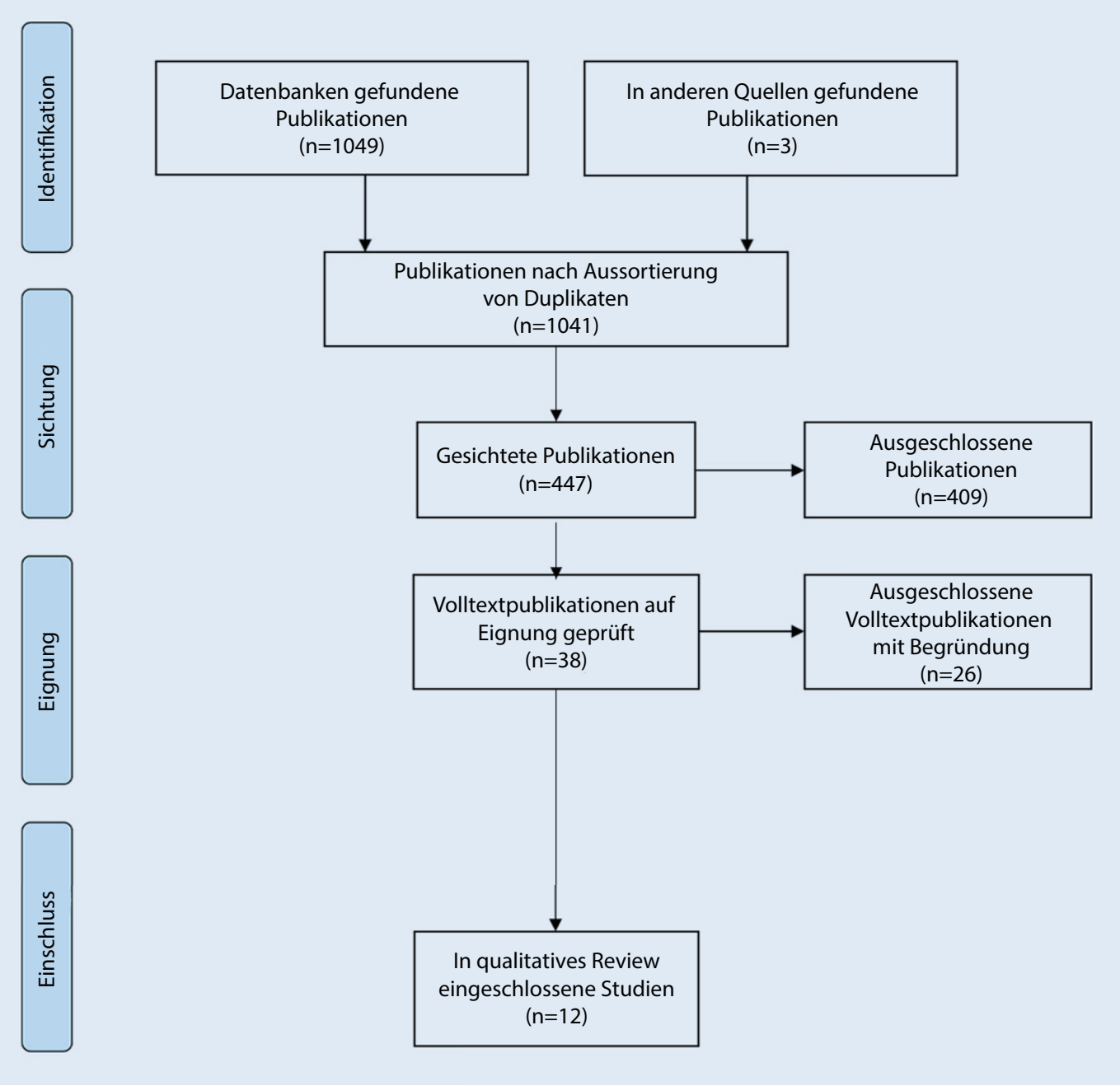

Abb. $1 \varangle$ Flowchart basierend auf dem PRISMA-Flussdiagramm

grationssituation steigt die Bedeutung direkter - insbesondere familiärer - Kontakte. Die Angst, das Gesicht zu verlieren oder die Familie in ein schlechtes Licht zu rücken, kann daher ausgeprägter sein [5-8, 18, 20]. Dies kann dazu führen, dass Betroffene es vorziehen, die Probleme selber oder familienintern $\mathrm{zu}$ lösen [5, 7-9, 18, 22, 29].

Eng mit dem Problem der Scham und Stigmatisierung verbunden ist die $\mathrm{Be}$ fürchtung, als „psychisch krank“ abgestempelt zu werden [20]. Erschwerend kommt hinzu, dass viele Migrantinnen und Migranten die glücksspielbedingte Störung nicht mit einer Sucht assoziieren $[2,6,9,20,22]$. Aufgrund der in der in der Regel kurzen Verweildauer bekunden Migrantinnen und Migranten zudem oftmals Schwierigkeiten, sich im Sozial- und Gesundheitswesen der Aufnahmegesellschaft zu orientieren bzw. wissen nicht, dass es solche Angebote gibt [2, 5-8, $20,22,25,28,29]$. Informationsdefizite können dazu führen, dass das Verständnis für „westliche“ psychologische Beratungs- und Behandlungskonzepte fehlt $[2,6,8,18,22,28,29]$. Dies wiederum kann zur Folge haben, dass sich Betroffene nicht in den Behandlungsprozess einbringen. Der Mangel an Vertrauen in die Konzepte führt dazu, dass Betroffene sehr zurückhaltend sind, im Rahmen einer Selbsthilfegruppe oder einer Beratungssituation mit Dritten über persönliche Probleme zu sprechen $[8,18,20]$. Skepsis kommt nicht nur gegenüber den Beratungs- und Behandlungskonzepten zum Ausdruck, sondern auch in Bezug auf Bedenken hinsichtlich Privatsphäre und Vertraulichkeit [5-7, 20]. Beratungsund Behandlungsangebote werden möglicherweise auch deshalb nicht genutzt, weil die Betroffenen negative Konsequen- zen für sich und ihre Familie befürchten. Das Misstrauen gegenüber Fachpersonen kann auch Ausdruck von erlebten Diskriminierungen sein [18]. Sprachliche Probleme und soziokulturell bedingte Missverständnisse sind ebenfalls $\mathrm{Zu}$ gangsbarrieren [2, 6-9, 20, 22]. Die Gründe für die geringe Inanspruchnahme der präventiven Angebote durch Migrantinnen und Migranten dürfen jedoch keineswegs einseitig bei der betroffenen $\mathrm{Be}$ völkerungsgruppe gesucht werden. Vielerorts lässt sich eine mangelhafte $\mathrm{Ab}$ stimmung des Gesundheitssystems auf die Bedürfnisse und den kulturellen Bezugsrahmen von Menschen mit Migrationshintergrund feststellen $[2,8,9,22$, 29]. 
Präv Gesundheitsf 2021 · 16:263-268 https://doi.org/10.1007/s11553-020-00815-8

(c) Der/die Autor(en) 2020

\section{S. Lischer}

\section{Die Inanspruchnahme von präventiven Angeboten durch Migrantinnen und Migranten mit glücksspielspezifischen Problemen}

\section{Zusammenfassung}

Ausgangslage. Personen mit Migrationshintergrund nehmen keineswegs öfter an Glücksspielen teil als die einheimische Bevölkerung, es ist aber wahrscheinlich, dass eine gleich häufige Teilnahme an Glücksspielen eher zu glücksspielbedingten Problemen führt.

Zielsetzung. Es wird untersucht, welche Zugangsbarrieren für Migrantinnen und Migranten mit glücksspielbedingten Problemen in Bezug auf die Inanspruchnahme von präventiven Angeboten bestehen und mit welchen Strategien die Wahrscheinlichkeit der Inanspruchnahme ebendieser Angebote erhöht werden kann.

Methode. Die narrative Literaturrecherche umfasst Studien und Übersichtsartikel, die zwischen 2014 und 2019 publiziert wurden.
Ergebnisse. Faktoren, welche Zugangsbarrieren darstellen können, sind Scham, die Angst vor Stigmatisierung und der Wunsch, das Problem selber oder im Kreis der Familie zu lösen. Hinzu kommt, dass glücksspielbedingte Probleme nicht mit Sucht assoziiert werden. Neben sprachlichen Problemen stellen die mangelnde Vertrautheit mit dem Gesundheitssystem sowie das fehlende Verständnis für die angewendeten Konzepte Hürden dar. Vielerorts lässt sich zudem eine mangelhafte Abstimmung des Gesundheitssystems auf die Bedürfnisse der Migrantinnen und Migranten feststellen. Um die Hürden gegenüber präventiven Angeboten zur Reduktion glücksspielbedingter Probleme zu senken, bedarf es eines stärkeren Einbezugs der anvisierten Zielgruppe.
Schlussfolgerungen. In Anlehnung an die Ottawa-Charta müssen partizipative Ansätze weiterentwickelt werden. Je frühzeitiger die Zielgruppen an der Problemdefinition und an der Gestaltung der präventiven Angebote partizipieren, desto niedriger sind die Barrieren, die der Inanspruchnahme dieser Angebote und einer Veränderung von gesundheitsbezogenen Verhaltensweisen entgegenstehen.

Schlüsselwörter

Glücksspielsucht · Barrieren · Suchtprävention - Erreichbarkeit · Muttersprachliche Präventionsangebote

\section{Utilization of preventive offers by migrants with gambling-related problems}

\section{Abstract}

Background. People with a migration background are less likely to gamble than nonmigrants but are more likely to experience problems from their gambling or be at risk of developing problems.

Objective. This literature review aims to identify the barriers to access for migrants with gambling-related problems. Particular focus will be given to their use of preventive services and strategies that can be used to increase the likelihood of utilizing service support.

Methods. The narrative literature review includes studies and review articles published between 2014 and 2019.
Results. Barriers that lead to the non-use of prevention services include embarrassment, stigma, the wish to handle problem on own or within the family, and the lack of association of gambling-related problems with addiction. In addition to language problems, the lack of familiarity with the health care system as well as the lack of understanding of the applied concepts represent barriers to access. Often, the health care system is also inadequately adapted to the needs of migrants. In order to decrease access barriers for preventive offers to reduce gambling-related problems, stronger involvement of the target group is needed.
Conclusions. In line with the Ottawa Charter, participatory approaches must be further developed. The earlier the target groups participate in the defining the problem and selecting preventive services, the lower the barriers to using these services and to changing health-related behavior patterns.

\section{Keywords}

Gambling addiction · Barriers · Addiction prevention - Reachability · Native speaking prevention offers

\section{Ansätze und Strategien für eine erhöhte Inanspruchnahme der präventiven Angebote}

Ansätze, welche auf die Minimierung von Scham und Angst vor Stigmatisierung abzielen, empfehlen die $\mathrm{Zu}$ sammenarbeit mit Multiplikatoren aus Migrationsgesellschaften, in denen die Prävalenz an glücksspielbedingten Problemen nachweislich besonders hoch ist. Die Strategie zielt darauf ab, dass diese Personen oder Institutionen die anvisierten Zielgruppen über die mit dem Glücksspiel verbundenen Risiken informieren und das Thema der Suchterkrankung ansprechen $[6,8,9,28]$. Die Beratungs- und Behandlungsangebote sollen bekannt gemacht und insbesondere entstigmatisiert werden $[6,8,9$, 20]. Weitere operative Empfehlungen legen nahe, dass Informationsbroschüren für pathologisch Glücksspielende abseits der Spielstätten und möglichst im öffentlichen Raum aufgelegt werden sollten, da diese von den Betroffenen aus
Scham vor Ort nur selten mitgenommen würden [20]. Ferner sollten spezifischen Informationen die Zugangswege zu entsprechenden Stellen erleichtern [18, 28]. Für das Bekanntmachen der präventiven Angebote muss aktiv auf die jeweiligen Migrationsgemeinden zugegangen werden $[6,7,18,22,28]$.

Für die Vermittlung von präventiven Botschaften darf der Zugang über die konventionellen Kanäle wie beispielsweise Informationsbroschüren nicht außer Acht gelassen werden. Am einfachsten 
Tab. 1 Studien zur Inanspruchnahme von präventiven Angeboten durch Migrantinnen und Migranten mit glücksspielbedingten Problemen

\begin{tabular}{|c|c|c|}
\hline Studie & Land & Methode \\
\hline [2] & Deutschland & Repräsentative Bevölkerungsstichprobe $(n=444)$ \\
\hline [5] & Australien & Narrative Literaturrecherche \\
\hline [6] & Kanada, Ontario & $\begin{array}{l}\text { Qualitative Interviews mit } n=5 \text { Fachstellenmitarbeitenden } \\
\text { sowie mit } n=5 \text { Mitarbeitenden eines "responsible gaming re- } \\
\text { source centres" }\end{array}$ \\
\hline [7] & Australien & $\begin{array}{l}\text { Leitfadengestützte Interviews mit Schlüsselpersonen }(n=18) \text {, } \\
\text { Einzelgespräche und Fokusgruppen mit chinesischen }(n=25) \\
\text { und tamilischen ( } n=11) \text { Migrantinnen und Migranten sowie } \\
\text { schriftliche Befragung aller Teilnehmenden }\end{array}$ \\
\hline [8] & Australien & Narrative Literaturrecherche \\
\hline [9] & Australien & $\begin{array}{l}\text { Repräsentative Bevölkerungsstudie (Querschnitt), } \\
\text { Personen mit und ohne problematischem Spielverhalten } \\
(n=730), \\
\text { davon } 34 \%(n=208) \text { nicht in Australien geboren }\end{array}$ \\
\hline [18] & Australien & Narrative Literaturrecherche und teilnehmende Beobachtungen \\
\hline [20] & Deutschland & $\begin{array}{l}\text { Leitfadengestützte Interviews mit Fachpersonen }(n=9) \text {, } \\
\text { leitfadengestützte Interviews mit türkischen Männern mit PG } \\
(n=8) \text {, } \\
\text { quantitative Befragung } n=81 \text { Männer mit PG, } n=80 \text { Männer } \\
\text { ohne PG }\end{array}$ \\
\hline [22] & International & Narratives Review \\
\hline [24] & USA & Narratives Review \\
\hline [28] & Neuseeland & Formative Evaluation, Durchführung mit Mixed-methods-Ansatz \\
\hline [29] & International & Systematisches Review ( $n=38$ Artikel) \\
\hline
\end{tabular}

erscheint es, Informationsmaterial $\mathrm{zu}$ übersetzen. Allerdings kann die reine sprachliche Übersetzung ohne inhaltliche Anpassung an Normen und Werte dazu führen, dass das Angebot als kulturell unangemessen wahrgenommen wird [3]. Dieses Problem stellt sich in besonderem Maße, weil Glücksspiele unterschiedlich konnotiert werden. Es empfiehlt sich daher, mit Mediatoren zusammenzuarbeiten, welche die Inhalte nicht „nur“ sprachlich, sondern auch in Bezug auf den soziokulturellen Bedeutungsgehalt vermitteln können $[6,9,18$, $22,28]$. Wird eine größere Reichweite angestrebt, müssen die Botschaften über spezifische Kanäle vermittelt werden. Dabei sollten die von den jeweiligen Migrationsgemeinden genutzten Medien berücksichtigt werden, etwa spezielle Radiosendungen, Zeitungen oder Social Media. Außerdem sind Settings wie Imbissstuben, Kulturlokale, aber auch Wartezimmer von ärztlichen Praxen für die Verbreitung von präventionsrelevanten Informationen $\mathrm{zu}$ nutzen $[8,9,18$, 22, 28].
Neben Maßnahmen, welche der primären Prävention und der Gesundheitsförderung zugeordnet werden können, bedarf es für Personen mit einer subklinischen Symptomatik Maßnahmen der sekundären Prävention. Viele Migrantinnen und Migranten sind eher bereit, mit Allgemeinpraktikerinnen und -praktikern über ihre Probleme zu sprechen [2, $8,22,24]$. Daher ist es wichtig, dass diese für die Thematik rund um das problematische Glücksspiel sensibilisiert sind. Neben Hausärztinnen und -ärzten sollten Fachpersonen der Sozialen Arbeit, aber auch Lehrpersonen für die Früherkennung von glücksspielbedingten Problemen geschult werden [15].

Neben der interkulturellen Kompetenz der Fachperson [8, 18, 29], welche unabdingbar ist, besteht das Bedürfnis nach muttersprachlicher Beratung und Behandlung [5-9, 18, 20, 29]. Es ist grundsätzlich erstrebenswert, dass Fachpersonen mit Migrationserfahrung für Präventions- und Behandlungsangebote qualifiziert werden. Ein solcher Hintergrund ist an und für sich ohne
Zweifel bereits eine Ressource; das gezielte Einsetzen dieser Ressource muss aber ebenfalls gelernt sein [6-9, 18, 22]. Steht kein muttersprachliches Personal zur Verfügung, sollten Dolmetscher zur Verfügung gestellt werden. Angehörige oder nähere Bekannte mit dieser Aufgabe zu betrauen, gilt gemeinhin als kritisch [20]. Da die Familie in Migrationssituationen eine wichtige Rolle spielen kann, müssen Behandlungskonzepte entwickelt werden, die auf Wunsch der Betroffenen Familienmitglieder einbeziehen [7, 9, 18, 22].

Es gibt Behandlungsansätze, welche sich explizit für die Anwendung in diversen Settings gut eignen. Kurze und kompakte Interventionen wie „motivational interviewing " oder die kognitive Verhaltenstherapie erweisen sich gerade auch vor dem Hintergrund, dass Migrantinnen und Migranten möglichweise einen niedrigeren sozioökonomischen Status haben als die einheimische Bevölkerung, als zielführend [24]. In Anbetracht dessen, dass sich Migrantinnen und Migranten in einem höheren Ausmaß vor Stigmatisierungen fürchten, erweisen sich online verfügbare Selbsthilfeprogramme als zielführend ([8]; • Tab. 2).

\section{Diskussion}

Die Sichtweise, dass aktiv auf die Migrationsbevölkerung zugegangen werden und mit Multiplikatoren zusammengearbeitet werden muss, hat sich in Theorie und Praxis durchgesetzt. Nichtsdestotrotz fällt auf, dass die in den Ergebnissen vorgeschlagenen Konzepte weitgehend von qualifizierten Expertinnen und Experten entwickelt wurden und damit mehrheitlich der Top-down-Sichtweise folgen. Ansätze, die der Bottom-upStrategie folgen, sollten in der Prävention gleichberechtigt verankert werden. Je frühzeitiger die Zielgruppen an der Problemdefinition und Angebotsgestaltung partizipieren, desto niedriger sind die Barrieren der Inanspruchnahme und Veränderung von gesundheitsbezogenen Verhaltensweisen [1]. Menschen können ihr Gesundheitspotenzial nur dann weitgehend selbst entfalten, wenn sie auf Faktoren, die ihre Gesundheit beein- 
Tab. 2 Ansätze und Strategien zur Reduktion von Zugangsbarrieren im Präventionsbereich

\section{Zugangsbarrieren}

Scham und Angst vor Stigmatisierung

$[5-10,18,20,22,24,29]$

Mangelnde Orientierung im Sozial- und Gesundheitssystem

$[2,5-8,20,22,25,28,29]$

Fehlendes Verständnis für die angewendeten Beratungsund Behandlungsmethoden $[2,6,8,18,22,28,29]$

Wunsch, das glücksspielbedingte Problem selber, oder im Kreis der Familie zu lösen $[5,7-9,18,22,29]$

Fehlende Assoziierung der glücksspielbedingten Störung mit einer Sucht

$[2,6,9,20,22]$

Sprachliche Barrieren und kulturell bedingte Missverständnisse

$[2,6-9,20,22]$

Mangelhafte Abstimmung des Gesundheitssystems auf die Bedürfnisse von Menschen mit Migrationshintergrund $[2,8,9,22,29]$

Bedenken hinsichtlich der Privatsphäre und der Vertraulichkeit

[5-7, 20]

Misstrauen aufgrund von erfahrener Diskriminierung [18]

\section{Ansätze und Strategien}

Einbezug der Migrationsbevölkerung

- Zusammenarbeit mit Multiplikatoren (Problemdefinition, Bedürfnisse), [6-9, 18, 20, 22, 22, 28]

- Übersetzung der Informationsbroschüren: Mediatoren einbeziehen $[3,6,9,18,22,28]$

- Nutzung der Medien der jeweiligen Migrationsbevölkerung $[8,9,18,22,28]$
- Einsatz von muttersprachlichen Fachpersonen [5-9, 18, 20, 22, 29]

- Dolmetscher $[8,20]$

- Interkultureller Kompetenz der Fachpersonen [8, 9, 18, 29]

- Sensibilisierung Hausärzt/innen und Sozialarbeitende für Glücksproblematik [2, 8, 22, 24]

- Behandlungskonzepte, die Familienmitglieder einbeziehen $[7,9,19,22]$

- Motivational Interviewing oder die kognitive Verhaltenstherapie [24]

- Online verfügbare Selbsthilfeprogramme [8]

- Förderung der Qualifikation von Fachpersonen mit Migrationserfahrung

Beratungs- und Behandlungsangebote,

- Zusammenarbeit mit Personen oder Institutionen, die die präventiven Botschaften an die Zielgruppe weiterleiten und dadurch multiplizieren,

- Förderung der Qualifikation von Fachpersonen mit Migrationserfahrung aus dem Gesundheitssystem.

\section{Früherkennung:}

- Der Zugang zur Regelversorgung ist einfacher; die Barrieren sind niedriger. Daher sollte diese Berufsgruppen für die Thematik des problematischen Glücksspiels sensibilisiert werden.

\section{Beratung und Behandlung:}

- muttersprachliche Behandlungsangebote,

- Behandlungskonzepte, die den Einbezug von Familienmitgliedern miteinschließen,

- Interventionen wie „motivational interviewing" oder die kognitive Verhaltenstherapie.

\section{Korrespondenzadresse}

\section{Prof. Dr. Suzanne Lische}

Hochschule Luzern, Soziale Arbeit

Werftestrasse 1, 6002 Luzern, Schweiz

suzanne.lischer@hslu.ch

Funding. Open access funding provided by Lucerne University of Applied Sciences and Arts

\section{Einhaltung ethischer Richtlinien}

[23].

In methodischer Hinsicht muss auf einige Begrenzungen hingewiesen werden. Die Untersuchung des Zusammenhangs zwischen Migrationshintergrund und glücksspielbedingten Problemen erweist sich als schwierig, weil die fundierte Analyse aller Verursachungsfaktoren von glückspielbedingten Problemen aufgrund ihrer Vielfältigkeit, auch ohne die Berücksichtigung von Migration, nahezu unüberschaubar erscheint [26]. Außerdem sind die Gründe, weshalb Betroffene Unterstützung annehmen bzw. nicht annehmen, in der Realität viel komplexer und vielfältiger. Die im Rahmen der analytischen Epidemiologie behandelte Frage, ob eine Assoziation besteht zwi- schen einem vermuteten Risikofaktor z. B. Sprache als Zugangsbarriere - und dem Outcome - (k)eine Inanspruchnahme einer Behandlung - und ob diese Assoziation kausal ist, kann mit den gängigen sozialwissenschaftlichen Methoden nicht überprüft werden. Die Ergebnisse müssen aus dieser Perspektive diskutiert und mit Vorsicht interpretiert werden.

\section{Fazit für die Praxis}

Prävention und Gesundheitsförderung:

- Einbezug der anvisierten Zielgruppe bei der Problemdefinition, der Bedürfnisartikulation und bei der Angebotsentwicklung,

- direkte Ansprache der Zielgruppe und Information über bestehende
Interessenkonflikt. S. Lischer gibt an, dass kein Interessenkonflikt besteht.

Für diesen Beitrag wurden von der Autorin keine Studien an Menschen oder Tieren durchgeführt Für die aufgeführten Studien gelten die jeweils dort angegebenen ethischen Richtlinien.

Open Access. Dieser Artikel wird unter der Creative Commons Namensnennung 4.0 International Lizenz veröffentlicht, welche die Nutzung, Vervielfältigung Bearbeitung, Verbreitung und Wiedergabe in jeglichem Medium und Format erlaubt, sofern Sie den/die ursprünglichen Autor(en) und die Quelle ordnungsgemäß nennen, einen Link zur Creative Commons Lizenz beifügen und angeben, ob Änderungen vorgenommen wurden.

Die in diesem Artikel enthaltenen Bilder und sonstiges Drittmaterial unterliegen ebenfalls der genannten Creative Commons Lizenz, sofern sich aus der Abbil- 


\section{Originalarbeit}

dungslegende nichts anderes ergibt. Sofern das betreffende Material nicht unter der genannten Creative Commons Lizenz steht und die betreffende Handlung nicht nach gesetzlichen Vorschriften erlaubt ist, ist für die oben aufgeführten Weiterverwendungen des Materials die Einwilligung des jeweiligen Rechteinhabers einzuholen.

Weitere Details zur Lizenz entnehmen Sie bitte der Lizenzinformation auf http://creativecommons.org/ licenses/by/4.0/deed.de.

\section{Literatur}

1. Altgeld T, Kickbusch I (2012) Gesundheitsförderung und Prävention. Public health. Gesundh Gesundheitswes 3:187-196

2. Bischof A, Meyer C, Bischof G et al (2015) Inanspruchnahme von Rehabilitationsleistungen bei pathologischen Glücksspielern. Suchttherapie 16:9-17

3. BrandT, Kleer D, Samkange-Zeeb F et al (2015) Prävention bei Menschen mit Migrationshintergrund. Bundesgesundheitsblatt Gesundheitsforschung Gesundheitsschutz58:584-592

4. Braun B, Ludwig M, Sleczka P et al (2014) Gamblers seeking treatment: who does and who doesn't? JBehav Addict 3:189-198

5. Dickins M, Thomas AC (2016) Gambling in Culturally and Linguistically Diverse Communities in Australia. Australian Gambling Research Centre, Australian Institute of Family Studies, Melbourne

6. Dinshaw FM, Mooney E, Pietropaolo V et al (2017) Exploring how immigrant and ethnocultural populations in southern ontario seek help for gambling problems

7. Feldman S, Radermacher H, Anderson Cetal (2014) A qualitative investigation of the experiences, attitudes and beliefs about gambling in the Chinese and Tamil communities in Victoria. Victorian Responsible Gambling Foundation Victoria, Victoria

8. Gainsbury S, Hing N, Suhonen N (2014) Professional help-seeking for gambling problems: awareness, barriers and motivators for treatment. J Gambl Stud 30:503-519

9. Gainsbury SM (2017) Cultural competence in the treatment of addictions: theory, practice and evidence. Clin Psychol Psychother 24:987-1001

10. Hing N, Russell A, Nuske E et al (2015) The stigma of problem gambling: causes, characteristics and consequences

11. Hodgins DC, El-Guebaly N (2000) Natural and treatment-assisted recovery from gambling problems: a comparison of resolved and active gamblers. Addiction 95:777-789

12. Jacoby $\mathrm{N}$, Von Lersner $\mathrm{U}$, Schubert $\mathrm{HJ}$ et al (2013) The role of acculturative stress and cultural backgrounds in migrants with pathological gambling. Int Gambl Stud 13:240-254

13. Johansson A, Grant JE, Kim SW et al (2009) Risk factors for problematic gambling: a critical literature review. J Gambl Stud 25:67-92

14. Kastirke N, Rumpf H-J, John U et al (2015) Demographicriskfactorsand gambling preference may not explain the high prevalence of gambling problems among the population with migration background: results from a German nationwide survey. J Gambl Stud 31:741-757

15. Lischer S, Häfeli J, Villiger S (2014) Sensibilisierung der Fachpersonen des externen Versorgungssys- tems für glücksspielsuchtspezifische Probleme. Sucht 60:289-296

16. Lischer S, Häfeli J, Villiger S (2014) Vulnerable Personengruppen im Glücksspielbereich. Präv Gesundheitsf 9:47-51

17. Loy JK, Grüne B, Braun B et al (2019) Help-seeking behaviour ofproblem gamblers: a narrative review. Sucht 64(5-6):259-272

18. Mazbouh-Moussa R, Ohtsuka K (2017) Cultural competence in working with the Arab Australian community: a conceptual review and the experience of the Arab Council Australia (ACA) gambling help counselling service. Asian J Gambl Issues Public Health 7:10

19. Meyer C, Rumpf H-J, Kreuzer A et al (2011) Pathologisches Glücksspielen und Epidemiologie (PAGE): Entstehung, Komorbidität, Remission und Behandlung. Endbericht an das Hessische Ministerium des Innern und für Sport. Universitäten Greifswald und Lübeck, Greifswald/Lübeck

20. Milin S, Buth S, Karakuş D et al (2017) Glücksspielprobleme bei türkeistämmigen Migranten Ursachen, Barrieren der Inanspruchnahme von Hilfen und Unterstützungsbedarfe. ISD-Hamburg, Hamburg

21. Moher D, Liberati A, Tetzlaff J et al (2009) Preferred reporting items for systematic reviews and metaanalyses: the PRISMA statement. PLoS Med 6:e1000097

22. Oei T, Raylu N, Loo J (2019) Roles of culture in gambling and gambling disorder. In: Gambling disorder. Springer, Berlin Heidelberg, S271-295

23. Organization WH (1993) Ottawa-Charta zur Gesundheitsförderung. WHO-autorisierte Übersetzung: Hildebrandt H, Kickbusch I (2020) WHORegionalbüro für Europa, Kopenhagen, S 8-13. https://www.euro.who.int/_data/assets/pdf_ file/0006/129534/Ottawa_Charter_G.pdf. Zugegriffen: 15. Mai 2020

24. Richard K, Baghurst T, Faragher JM et al (2017) Practical treatments considering the role of sociocultural factors on problem gambling. J Gambl Stud 33:265-281

25. Rommel A, Köppen J (2016) Migration und Suchthilfe - Inanspruchnahme von Leistungen durch Menschen mit Migrationshintergrund. Psychiat Prax 43:82-88

26. Sahrai D (2009) Healthy Migrants oder besondere Risikogruppe. Jahrb Krit Med Gesundheitswiss 45:70-94

27. Suurvali H, Cordingley J, Hodgins DC et al (2009) Barriers to seeking help for gambling problems: a review of the empirical literature. J Gambl Stud 25:407-424

28. Tse S, Wong J, Lim G et al (2014) "Gamblefree day," A community project to minimise harm caused by gambling disorder in new zealand Chinese and Korean communities: formative evaluation and lessons learnt. J Psychosoc Rehabil Ment Health 1:77-84

29. Wardle H, Bramley S, Norrie C et al (2019) What do we know about gambling-related harm affecting migrants and migrant communities? A rapid review. Addict Behav 93:180-193 